\title{
ON THE SPECTRUM OF $\alpha$-RIGID MAPS
}

\author{
E. H. EL ABDALAOUI
}

\begin{abstract}
It is shown that there exists an $\alpha$-rigid transformation with $\alpha$ less or equal to $\frac{1}{2}$ whose spectrum has Lebesgue component. This answers the question raised by Klemes and Reinhold in [30]. Moreover, we introduce a new criterium to identify a large class of $\alpha$-rigid transformations with singular spectrum.
\end{abstract}

\section{INTRODUCTION}

In his 1980's paper [27], A. Katok showed that interval exchange maps are not mixing. As a consequence of Katok's proof interval exchange maps are $\alpha$-rigid. In a later work, Veech in [43] investigated the spectral properties of interval exchange maps and showed that almost every minimal interval exchange maps has singular simple spectrum. Before, Oseledets in [21] proved that for any interval exchange transformation the maximal spectral multiplicity is bounded above by $r-1$, where $r$ is the number of intervals exchanged. Moreover, he constructed the first example of a transformation with continuous spectrum and finite multiplicity greater than 1 . Since the example is an exchange of 30 intervals, the maximal spectral multiplicity $m$ satisfies $2 \leq m \leq 29$. Robinson [35] constructed ergodic interval exchange transformations with arbitrary finite maximal spectral multiplicity. It follows from [28] that all the examples constructed by Oseledets and Robinson have singular spectrum. However Katok's theorem [27] remains at the present time the only universal result about the spectrum of interval exchange maps. Following Veech one may ask if there is some other "universal" spectral property satisfied by interval exchange maps. More precisely, as in [1] one may ask the following question.

Question 1. Does any interval exchange map have singular spectral type?

The answer to the above question is affirmative in the case of three interval exchange maps. Note that using the result in [17] one may show that every ergodic interval exchange transformation on three intervals has singular spectrum.

On the other hand, Klemes and Reinhold in [30] proved that for any $\alpha \in] 0,1[$, there exists an $\alpha$-rigid rank one transformation with singular spectrum and asked the following question.

Question 2. Does any $\alpha$-rigid transformation have singular spectral type?

It is well known that the answer is affirmative if $\alpha>\frac{1}{2}$ (Baxter's theorem). Note that if this was true for all $\alpha \leq 1$ then the answer the question 1 would be affirmative too.

2000 Mathematics Subject Classification.

Key words and phrases. $\alpha$-rigid transformation, interval exchange maps, Lebesgue spectrum, substitution, quasi-analyticity, joinings . 
In this paper we shall prove that this is not the case. More precisely, we prove in section 3 that the Mathew-Nadkarni transformation (which has Lebesgue component in the spectrum) is $\frac{1}{2}$-rigid. We recall that Mathew-Nadkarni introduced this transformation in 1984 in [19] to answer the question raised by Helson and Parry [18] of whether there exists an ergodic measure preserving transformation which has a Lebesgue component in its spectrum with finite non-zero multiplicity. Helson and Parry also mentioned the problem, attributed to Banach, whether there exists an ergodic measure preserving transformation on a finite measure space whose spectrum is simple Lebesgue. In [36], Rokhlin mentioned the problem of finding an ergodic measure preserving transformation on a finite measure space whose spectrum is of Lebesgue type with finite multiplicity. Another contribution to the Banach-Rohklin question is due to M. Queffélec [32] who proved that the spectrum of the Rudin-Shapiro substitution has Lebesgue component of multiplicity two. It turns out that the Rudin-Shapiro substitution is $\frac{1}{2}$-rigid.

In section 4 we will present other examples of dynamical systems arising from substitutions with Lebesgue component of multiplicity two whose rigidity constant is less than $\frac{1}{2}$.

It is an easy exercise to prove that $\alpha$-rigid transformations are not mixing. Since $\alpha$-rigidity does not imply that the spectrum is singular (as we shall see in the section 3), the absence of mixing does not imply that the spectrum is singular. Let us mention that any aperiodic measure-preserving transformation can be realized as an exchange of an infinite number of intervals [3].

Our work on the question of Klemes and Reinhold (question 2) includes a survey of the results of Dekking-Keane [8] and Queffélec [33]. F. M. Dekking and M. Keane showed that the dynamical systems arising from substitutions are not mixing. The ingredients of the proof lead to establish that the dynamical systems arising from substitutions are $\alpha$-rigid. The procedure to check the constant $\alpha$ will be presented in the last section without proof. Queffélec showed that the substitution which gives rise to the Rudin-Shapiro sequence has Lebesgue component of multiplicity two together with a discrete component. (Kamae [25] had earlier shown that the correlation measure of the Rudin-Shapiro sequence is Lebesgue).

In this section 2 we shall exhibit a large class of $\alpha$-rigid transformations with singular spectrum. More precisely, we shall prove that the transformation satisfying the restricted Beurling condition is singular. We say that the transformation $T$ satisfies the restricted Beurling condition if the following holds

$$
\begin{aligned}
& \left\{\sum_{i \in \mathbb{Z}} a_{i} U_{T}^{i}: a_{i}>0 \text { for some } i \text { and } \sum_{n \geq 0} \frac{\log \left(\sum_{k \leq-n} a_{k}^{2}\right)}{n^{2}}=-\infty\right\} \\
& \bigcap\left({\overline{\left\{U_{T}^{n}, n \in \mathbb{Z}\right\}}}^{W} \backslash\left(\left\{U_{T}^{n}, n \in \mathbb{Z}\right\}\right)\right) \neq \emptyset .
\end{aligned}
$$


Where $U_{T}$ is the operator defined by $U_{T} f(x)=f\left(T^{-1} x\right)$ and ${\overline{\left\{U_{T}^{n}, n \in \mathbb{Z}\right\}}}^{W}$ is the weak closure power of $T$.

We mention that the $\alpha$-rigid rank one transformations constructed by Klemes \& Reinhold statify the condition above. Thus, our result can be considered as a generalization of the Klemes-Reinhold result. There is another motivation for our work. In 1979 D. Rudolph [37] introduced the notion of minimal self-joinings as the foundation for a machinery that yields a wide variety of counterexamples in ergodic theory. But the property of minimal self-joinings is meager with respect to the weak topology on the group of the all automorphisms. Later, in $1983 \mathrm{~A}$. del Junco and M. Lemańczyk in [9] showed that these constructions, as well as many others in [37], can be based on a much weaker property that is in fact generic (residual in the weak topology). A special case of this property is the property of $\kappa$-mixing (see Katok [26], Stepin [42]) which implies the mutual singularity of the convolution powers of the maximal spectral type of an automorphism $T$. We remark also that any $\kappa$-mixing transformation is $(1-\kappa)$-rigid.

Question 3. Are the convolution powers of the maximal spectral type of any nonmixing transformation with minimal self-joinings property pairwise singular?

This would imply that the spectrum of any non-mixing transformation with minimal self-joinings is singular. In this paper we are not able to answer the question 3. Nevertheless we shall exhibit in section 2 a large class of non-mixing transformation with minimal self-joinings with singular spectrum.

Recently, some progress was obtained by Prikhod'ko and Ryzhikov in [24]. The authors show that the well-known Chacon transformation [7] possesses this property. It is well known that the Chacon transformation has the minimal self-joining property [11]. This result can be extended easily to the case of staircase transformations with bounded cutting parameter. The latter examples include as a special case the Klemes-Reinhold examples of $\alpha$-rigid rank one transformations.

We recall now some basic facts on spectral theory. A nice account can be found in the appendix of [22].

1.1. Spectral measures. Given a measure preserving invertible transformation $T: X \mapsto X$ and denoting as above by $U_{T} f$ the operator $U_{T} f(x)=f\left(T^{-1} x\right)$, for any $f \in L^{2}(X)$ there exists a positive measure $\sigma_{f}$ on the unit circle $S^{1}$ defined by $\hat{\sigma}_{f}(n)=<U_{T}^{n} f, f>$.

Definition . The maximal spectral type of $T$ is the equivalence class of Borel measures $\sigma$ on $\mathbb{T}$ (under the equivalence relation $\mu_{1} \sim \mu_{2}$ if and only if $\mu_{1}<<\mu_{2}$ and $\left.\mu_{2}<<\mu_{1}\right)$, such that $\sigma_{f}<<\sigma$ for all $f \in L^{2}(X)$ and if $\nu$ is another measure for which $\sigma_{f}<<\nu$ for all $f \in L^{2}(X)$ then $\sigma<<\nu$.

By the canonical decomposition of $L^{2}(X)$ into decreasing cycles with respect to the operator $U_{T}$, there exists a Borel measure $\sigma=\sigma_{f}$ for some $f \in L^{2}(X)$, such that $\sigma$ is in the equivalence class defining the maximal spectral type of $T$. By abuse of notation, we will call this measure the maximal spectral type measure. It can be replaced by any other measure in its equivalence class. The reduced maximal type 
$\sigma_{T}^{(0)}$ is the maximal spectral type of $U_{T}$ on $L_{0}^{2}(X) \stackrel{\text { def }}{=}\left\{f \in L^{2}(X): \int f d \mu=0\right\}$. The spectrum of $T$ is said to be discrete (resp. continuous, resp. singular, resp. absolutely continuous, resp. Lebesgue ) if $\sigma_{T}^{(0)}$ is discrete ( resp. continuous, resp. singular, resp. absolutely continuous with respect to the Lebesgue measure or equivalent to the Lebesgue measure). We write

$$
Z(h) \stackrel{\text { def }}{=} \overline{\operatorname{span}\left\{U_{T}^{n} h, n \in \mathbb{Z}\right\}} .
$$

The transformation $T$ is said to have simple spectrum, if there exists $h \in L^{2}(X)$ such that

$$
Z(h)=L^{2}(X) .
$$

Two dynamical systems $(X, \mathcal{A}, \mu, T)$ and $(Y, \mathcal{B}, \nu, S)$ are spectrally disjoint if $\sigma_{T}^{(0)}$ and $\sigma_{S}^{(0)}$ are mutually singular.

\section{2. $\alpha$-rigid transformations.}

Definition . A measure-preserving transformation $T$ on the probability space $(X, \mathcal{B}, \mu)$ is said to be $\alpha$-rigid, where $\alpha \in] 0,1]$, if there is a sequence of integers $\left\{n_{k}\right\}_{k \in \mathbb{N}}$ such that

$$
\lim _{k \rightarrow \infty} \mu\left(T^{n_{k}} A \cap A\right) \geq \alpha \mu(A), \forall A \in \mathcal{B} .
$$

The notion of $\alpha$-rigidity has been formulated in 1987 by N. Friedman [14]. Besides this, in $1969 \mathrm{~J}$. Baxter proved [5]

Theorem 4 (Baxter). The spectrum of any $\alpha$-rigid transformation is singular if $\alpha>\frac{1}{2}$.

Note that this theorem is a consequence of the following result

Theorem 5 (Ryzhikov[41]). Any $\alpha$-rigid transformation $T$ with $\alpha>\frac{1}{2}$ is spectrally disjoint from any mixing transformation $S$

Proof. By assumption, there exists a sequence of integers and a Markov operator $P$ such that $\left(U_{T}^{n_{i}}\right)$ converges weakly to $\alpha I+(1-\alpha) P$. Let $J$ be any operator such that

$$
U_{T} J=J U_{S}
$$

Then, for any $f \in L_{0}^{2}(X)$ we have

$$
\alpha J f+(1-\alpha) P J f=0 .
$$

Since the norm of $P$ is $\leq 1$, the operator $\alpha I+(1-\alpha) P$ is invertible. Hence, $J f$ must be 0 thus $J \equiv 0$. It follows that $T$ is spectrally disjoint from $S$ and the proof of the theorem is complete.

Remark 1 . The proof above gives more, namely if the weak closure of the powers of the operator $U_{T}$ contains any invertible operator, then $T$ is spectrally disjoint from any mixing maps. In fact, assume that there exists an invertible operator $V$ in the weak closure of the power of $T$ which means that there exists a sequence of integers $\left(n_{i}\right)$ such that

$$
\lim _{i \longrightarrow+\infty}\left(U_{T}^{n_{i}} f, f\right)=(V f, f),
$$


for any $f \in L_{0}^{2}(X)$. Let $S$ be any mixing map. By the Lebesgue decomposition of $\sigma_{T}^{(0)}$ with respect to the maximal spectral type of $S$ we have

$$
\sigma_{T}^{(0)}=\sigma_{s}+\sigma_{a} .
$$

It is well known that there exists a function $f \in L_{0}^{2}(X)$ such that $\sigma_{f}=\sigma_{a}$. But for any function $g \in L_{0}^{2}(X)$ we have

$$
\lim _{j \longrightarrow+\infty} \lim _{i \longrightarrow+\infty}\left(U_{T}^{n_{i}-n_{j}} g, g\right)=(V g, V g) .
$$

Applying the Riemann-Lebesgue theorem we get

$$
(V f, V f)=0,
$$

therefore $f=0$ and the proof is complete.

1.3. Joinings. Let $T$ be any ergodic automorphism of a Lebesgue space $(X, \mathcal{B}, \mu)$. The centralizer $C(T)$ of $T$ is the semi-group of all endomorphisms $S:(X, \mathcal{B}, \mu) \rightarrow$ $(X, \mathcal{B}, \mu)$ such that $S T=T S$. We say that $\lambda$ is a $n$-joining of $T$ if $\lambda$ is a $T^{\otimes n_{-}}$ invariant probability measure on $\mathcal{B}^{\otimes n}$ and $\lambda_{\mid \mathcal{B}}=\mu$, where $T^{\otimes n} \stackrel{\text { def }}{=} T \times T \cdots \times T$. Denote by $J(T, \cdots, T)$ the space of all $n$-joinings of $T$.

A standard example of ergodic 2-joinings comes from the centralizer of $T$. More precisely, if $S \in C(T)$ then the measure given by

$$
\Delta_{S}(A \times B)=\mu\left(A \cap S^{-1} B\right)
$$

is a 2-joining. For $S=T^{n}, n \in \mathbb{Z}$, we put $\Delta_{n} \stackrel{\text { def }}{=} \Delta_{T^{n}}$ and $\Delta \stackrel{\text { def }}{=} \Delta_{0}$.

Following [10] and [44], $T$ is called 2-fold simple if each ergodic 2-joining of $T$ is either on the graph of some $S \in C(T)$ or is the product measure $\mu \times \mu$. It is easy to see that 2-fold simplicity of $T$ implies that $C(T)$ is a group (consider $\left.\mu_{S}(A \times B)=\mu\left(S^{-1} A \cap B\right)\right)$.

Now, for $n>1$ and any $S_{i} \in C(T), i=1, \cdots, n$, the $n$-joining measure $\mu_{S_{1}, \cdots, S_{n}}$ given by

is said to be off-diagonal.

$$
\mu_{S_{1}, \cdots, S_{n}}\left(A_{1} \times \cdots \times A_{n}\right)=\mu\left(S_{1}^{-1} A_{1} \cap \cdots \cap S_{n}^{-1} A_{n}\right),
$$

The transformation $T$ is said to be simple if $C(T)$ is a group and for every $n \geq 2$ and every ergodic $n$-joining $\lambda$ the set $\{1, \cdots, n\}$ can be split into subsets $s_{1}, \cdots, s_{k}$ such that each $\left.\lambda\right|_{\mathcal{B}^{s_{i}}}$ is off-diagonal and $\lambda$ is their product [10]. If $T$ is 2 -fold simple and $C(T)$ is trivial, i.e., $C(T)=\left\{T^{i}: i \in \mathbb{Z}\right\}$, then $T$ is said to have the property of minimal self-joining of order $2(T \in M S J(2))$. Moreover $T$ is said to have minimal self-joinings of any order if $T$ is simple and $C(T)$ is trivial. The class of $M S J(2)$ transformations is contained in the class of transformations with finite joining rank. The joining rank of any ergodic transformation $T$, written jrk(T), is defined as the minimum of $r \in \mathbb{N}$ for which each ergodic $r$-fold self-joining $\nu$ has some pair $i<j$ such that the two-dimensional marginal $\nu_{\mathcal{B}^{(i)} \times \mathcal{B}^{(j)}}$ is trivial (i.e., $\nu_{\mathcal{B}^{(i)} \times \mathcal{B}^{(j)}} \in\{\mu \times \mu\} \cup\left\{\Delta_{n}\right\}_{n \in \mathbb{Z}}$ ). The standard examples of transformations with finite joining rank greater than 2 is given by the powers of any weak mixing transformation $T$ in $M S J(2)$. In addition, a non-mixing transformation with the minimal self-joining property is $\alpha$-rigid. Indeed, we have the following 
Lemma 1 ([38]). Let $T$ be a non-mixing map in $M S J(2)$. Then $T$ is $\alpha$-rigid for some $\alpha \in] 0,1[$.

Proof. Since the set $J(T, T)$ is compact and $T$ is not mixing, there is a sequence $n_{k} \rightarrow \infty$ such that the sequence of diagonal measures $\Delta_{n_{k}}$ converges to some measure $\lambda \in J(T, T)$, where $\lambda \neq \mu \times \mu$. Since $T \in M S J(2)$, by the ergodic decomposition, we have

$$
\lambda=\beta \mu \times \mu+\sum_{i \in \mathbb{Z}} a_{i} \Delta_{i},
$$

where $a_{i} \geq 0$ and $\sum_{i \in \mathbb{Z}} a_{i}=1-\beta$. Hence, for some $i$ we have

$$
\lambda \geq a_{i} \Delta_{i}, a_{i}>0 .
$$

Thus, for the sequence $m_{k}=n_{k}-i$, we obtain

$$
\lim _{k \rightarrow \infty} \mu\left(T^{m_{k}} A \cap A\right) \geq \alpha \mu(A)
$$

where $\alpha \stackrel{\text { def }}{=} a_{i}$ and the proof of the lemma is complete.

Remark on the connection between joinning and Markov operators. It is well known and easy to see that for any joining $\lambda$ between two dynamical systems $(X, \mathcal{A}, T, \mu)$ and $(Y, \mathcal{B}, S, \nu)$ there exists a Markov operator $J: L_{0}^{2}(X, \mu) \longrightarrow$ $L_{0}^{2}(Y, \nu)$ such that

(1) $J \circ U_{T}=U_{S} \circ J$

(2) $J \chi_{X}=\chi_{Y}$ and $J^{*} \chi_{Y}=\chi_{X}$,

(3) $f>0$ and $g>0$ implies $J f>0$ and $J^{*} g>0$,

(4) for any $f \in L_{0}^{2}(X, \mu)$ and $g \in L_{0}^{2}(Y, \nu)$ we have

$$
\int_{X \times Y} f(x) g(y) d \lambda(x, y)=\int_{Y} J f(y) g(y) d \nu(y) .
$$

Here $J^{*}$ is the adjoint of $J$ defined by $(J f, g)=\left(f, J^{*} g\right)$ for $f \in L_{0}^{2}(X, \mu)$ and $g \in L_{0}^{2}(Y, \nu)$. The correspondence is one-to-one. The systems $(X, \mathcal{A}, T, \mu)$ and $(Y, \mathcal{B}, S, \nu)$ are disjoint (in the sens of Furstenberg) if $\mathrm{J}$ is trivial, where trivial means $J f=\int f d \mu$, for any $f \in L^{2}(X, \mu)$. To tackle some problems is ergodic theory using this approach is proposed for example in [39]. Using the same approach, FraçzekLemańczyk [12] show that the ergodic $\alpha$-rigid maps with $\alpha>0$ is disjoint from all mixing maps. Indeed, let $\lambda$ be any ergodic joining between a ergodic $\alpha$-rigid map $T$ and a mixing map $S$ and let $J$ be a associated Markov operator. By the $\alpha$-rigidity of $T$ it is easy to see that there is a sequence of integers $\left(n_{k}\right)_{k \in \mathbb{N}}$ and a Markov operator $P$ such that $\left(U_{T}^{n_{k}}\right)$ converges weakly to $\alpha I d+(1-\alpha) P$. Thus $J U_{T}^{n_{k}}$ converges weakly to $\alpha J+(1-\alpha) J P$. Since $J U_{T}^{n_{k}}=U_{S}^{n_{k}} J$ and $S$ is mixing we have $\left(U_{S}^{n_{k}} J f, g\right)=0$ for each $f \in L_{0}^{2}(X, \mu)$ and $g \in L_{0}^{2}(Y, \nu)$. As a consequence

$$
\alpha(J f, g)+(1-\alpha)(J P f, g)=0
$$

for each $f \in L_{0}^{2}(X, \mu)$ and $g \in L_{0}^{2}(Y, \nu)$. This gives, for all $f \in L^{2}(X, \mu)$,

$$
\alpha J f+(1-\alpha) J P f=\int f d \mu .
$$


Since the dynamical system $(X \times X, \mathcal{A} \otimes \mathcal{B}, T \times S, \mu \times \nu)$ is ergodic and $J P U_{T}=$ $U_{S} J P$, it follows that the operator $f \in L^{2}(X, \mu) \longmapsto \int f d \mu$ is indecomposable (i.e., is an extreme point of the subspace of Markov operators). Therefore $J f=\int f d \mu$ for each $f \in L^{2}(X, \mu)$ and the proof is complete.

In the following we shall establish the connection between the old problem of Banach on the existence of dynamical systems with simple Lebesgue spectrum and the $M S J$ property. For that, we recall the following conjecture.

Conjecture 6. ([22],pp. 50) If a reduced maximal spectral type of some transformation with simple spectrum is absolutely continuous then it is Lebesgue.

Theorem 7. If $T$ is a transformation with finite joining rank and simple Lebesgue spectrum then Conjecture 6 implies that $T$ is in the class MSJ.

Proof. By the King-Thouvenot theorem [29], $T$ is the $e$-extension $\left(e \in \mathbb{N}^{*}\right)$ of the power (say $p$ ) of some transformation $S$ with the $M S J$ property. But $S^{p}$ is a factor of $T$. It follows that $S^{p}$ and $S$ have simple Lebesgue spectrum. Since the multiplicity of $S^{p}$ is $p$ we have $p=1$. Thus $T$ is an $e$-extension of $S$. Now, by the classical spectral decomposition of the group extension we get $e=1$ and this concludes the proof of the theorem.

\section{MAin Result: BeuRling CONDition.}

In this section we introduce the following condition.

Definition . We say that the transformation $T$ satisfies the "Beurling condition " if

$$
\begin{aligned}
& \left\{\sum_{i \in \mathbb{Z}} a_{i} U_{T}^{i}: a_{i} \neq 0 \text { for some } i \text { and } \sum_{n \geq 0} \frac{\log \left(\sum_{k \leq-n} a_{k}^{2}\right)}{n^{2}}=-\infty\right\} \\
& \bigcap\left({\overline{\left\{U_{T}^{n}, n \in \mathbb{Z}\right\}}}^{W} \backslash\left(\left\{U_{T}^{n}, n \in \mathbb{Z}\right\}\right)\right) \neq \emptyset .
\end{aligned}
$$

This definition is inspired by early work of Beurling on a quasi-analytic class of mappings and on the uncertainty principle in harmonic analysis [4] combined by ideas coming from the joining theory.

The main result of this paper is the following theorem.

Theorem 8. If the transformation $T$ satisfy the Beurling condition then the spectrum of $T$ is singular.

To prove this theorem we shall need the following lemma. We recall that a measure $\nu$ on the circle is called a Rajchman measure if $\lim _{n \rightarrow \infty} \widehat{\nu}(n)=0$. 
Lemma 2 (Translation lemma). Suppose $\mu$ is a probability measure on $\mathbb{T}$ and $\left(n_{k}\right)_{k \in \mathbb{N}}$ is a sequence of distinct integers. Define $\mu_{k}$ by

$$
\mu_{k}=e^{i n_{k} \theta} d \mu(\theta), \quad k=1,2,3, \cdots
$$

If $\left(\mu_{k}\right)_{k \in \mathbb{Z}}$ converges in the weak ${ }^{*}$ topology to $\sigma$, then $\sigma$ is singular with respect to any Rajchman measure $\nu$. In fact, if $\mu=\mu_{s}+\mu_{a}$ is the Lebesgue decomposition of $\mu$ with respect to the Rajchman measure $\nu$ on $\mathbb{T}$, then

$$
|\sigma|(E) \leq \mu_{s}(E), \text { for every Borel set } E \text { in } \mathbb{T} .
$$

The proof of this lemma is the same as the proof given in $[34$, pp. 66] in the case of Lebesgue measure. We will also need the following Beurling theorem proved in [4]

Theorem 9 (Beurling's Theorem). Let $f$ be the function on torus $\mathbb{T}$ define by

$$
f(\theta)=\sum_{j \in \mathbb{Z}} a_{j} e^{i j \theta}
$$

and assume that the following condition holds

$$
\sum_{n \geq 0} \frac{\log \left(\sum_{k \leq-n} a_{k}^{2}\right)}{n^{2}}=-\infty .
$$

Then if $f=0$ on the set of positive measure we have $f=0$ a.e. on $\mathbb{T}$.

Proof. (of Theorem 8) Let $\sigma \stackrel{\text { def }}{=} \sigma_{h}$ be the reduced maximal spectral type of $T$. It follows from the Beurling condition that there exists a sequence of integers $\left(n_{k}\right)_{k \in \mathbb{N}}$ such that

$$
e^{i n_{k} \theta} d \sigma \stackrel{\mathrm{w} *}{\rightarrow} \sum_{j \in \mathbb{Z}} a_{j} e^{i j \theta} d \sigma .
$$

Let $\sigma=\sigma_{s}+\sigma_{a}$ be the Lebesgue decomposition of $\sigma$ with respect to the Lebesgue measure $m$ on $\mathbb{T}$. Put

$$
f(\theta) \stackrel{\text { def }}{=} \sum_{j \in \mathbb{Z}} a_{j} e^{i j \theta}
$$

Then, by the translation lemma, we have

$$
\sigma_{a}\{x: f(x)=0\}=\sigma_{a}(\mathbb{T}) .
$$

Now Beurling's theorem (Theorem 9) says that

$$
f \neq 0 \Rightarrow m\{x: f(x)=0\}=0 .
$$

Hence $\sigma_{a}=0$ and the proof of the theorem is completes.

As an immediate consequence of Theorem 8 we obtain the following 
Corollary 1. If the transformation $T$ satisfies the following restricted Beurling condition

$$
\begin{aligned}
& \left\{\sum_{i \in \mathbb{Z}} a_{i} U_{T}^{i}: a_{i}>0 \text { for some } i \text { and } \sum_{n \geq 0} \frac{\log \left(\sum_{k \leq-n} a_{k}^{2}\right)}{n^{2}}=-\infty\right\} \\
& \bigcap\left({\overline{\left\{U_{T}^{n}, n \in \mathbb{Z}\right\}}}^{W} \backslash\left(\left\{U_{T}^{n}, n \in \mathbb{Z}\right\}\right)\right) \neq \emptyset .
\end{aligned}
$$

Then $T$ is $\alpha$-rigid with singular spectrum.

Remark 2. Note that we have actually proved that $T$ is spectrally disjoint from any mixing maps $S$ provided that

$$
\sigma_{S}^{(0)}(\{x: f(x)=0\})=0 .
$$

Observe that (2.4) holds if $f$ is an analytic function on the circle.

2.1. Applications. Using Theorem 8 we shall give in this section a simple proof of some well known results on the singularity of the spectrum of some special maps of rank one maps. More precisely, we shall give a simple proof of the singularity of Chacon maps and the staircase maps with bounded cutting parameter. Recall that for any $\varepsilon>0$ one may construct a staircase maps with bounded cutting parameter, say $p$, such that the $\alpha$-rigid constant is smaller that $\varepsilon$. In fact, the $\alpha$-rigid constant is $\frac{1}{p}$.

Let us recall the definition of rank one maps and in particular the staircase maps. Using the cutting and stacking method described in [15], [16], one can define inductively a family of measure preserving transformations, called rank one transformations, as follows.

Let $B_{0}$ be the unit interval equipped with the Lebesgue measure. At stage one we divide $B_{0}$ into $p_{0}$ equal parts, add spacers and form a stack of height $h_{1}$ in the usual way. At the $k^{t h}$ stage we divide the stack obtained at the $(k-1)^{t h}$ stage into $p_{k-1}$ equal columns, add spacers and obtain a new stack of height $h_{k}$. If during the $k^{\text {th }}$ stage of our construction the number of spacers put above the $j^{\text {th }}$ column of the $(k-1)^{t h}$ stack is $a_{j}^{(k-1)}, 0 \leq a_{j}^{(k-1)} \leq \infty, 1 \leq j \leq p_{k}$, then we have

$$
h_{k}=p_{k-1} h_{k-1}+\sum_{j=1}^{p_{k-1}} a_{j}^{(k-1)} .
$$

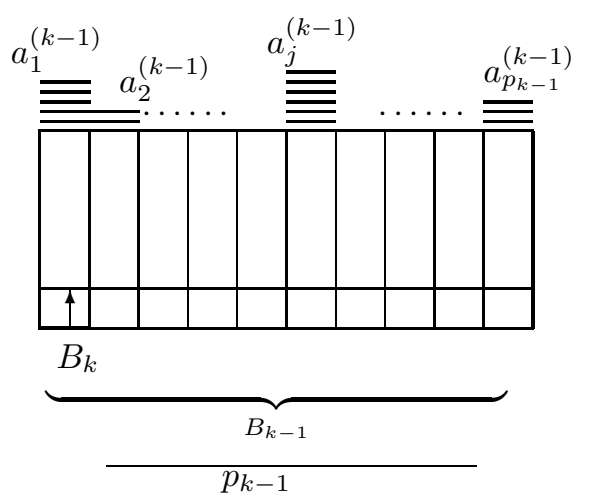

Figure $1: k^{\text {th }}$-tower. 
Proceeding in this way, we get a rank one transformation $T$ on a certain measure space $(X, \mathcal{B}, \nu)$ which may be finite or $\sigma$-finite depending on the number of spacers we added.

The construction of any rank one transformation thus needs two parameters: $\left(p_{k}\right)_{k=0}^{\infty}$ (cutting and stacking parameter), and $\left(\left(a_{j}^{(k)}\right)_{j=1}^{p_{k}}\right)_{k=0}^{\infty}$ (spacers parameter). Let

$$
T \stackrel{\text { def }}{=} T_{\left(p_{k},\left(a_{j}^{(k)}\right)_{j=1}^{\left.p_{k}\right)}\right)_{k=0}^{\infty}} .
$$

In the case of staircase maps the spacers parameter is given by

$$
a_{j}^{(k)}=j-1, \text { for } j=1 \ldots p_{k}-1 \text { and } a_{p_{k}}^{(k)}=0 .
$$

The classical Chacon map [7] corresponds to the case $p_{k}=3$, for every $k \in \mathbb{N}^{*}$. It is easy to see that the Chacon map is $\frac{1}{3}$-rigid. More generally, it is easy to prove that the staircase with bound cutting parameter (say $p$ ) is $\frac{1}{p}$-rigid (in fact, for any measurable set $A$ we have $\left.\liminf \mu\left(T^{h_{n}} A \cap A\right) \geq \frac{1}{p} \mu(A)\right)$. The following lemma can be may proved using Theorem 8 and the Remark 2. Nevertheless for the convenience of the reader we present a different proof.

Lemma 3. Let $T$ be a weak mixing transformation and assume that there is a sequence of integers $\left(n_{k}\right)$ such that $\left(U_{T}^{n_{k}}\right)_{k \in \mathbb{N}}$ converges weakly to $P(T)$, where $P(z)$ is a nonzero analytic function on the circle. Then $T$ is spectrally disjoint from any mixing transformation $S$.

Proof. (Ryzhikov [41],[12]) Let $J$ be any operator such that

$$
U_{T} J=J U_{S} .
$$

Then $P(T) J f=0$ for each $f \in L_{0}^{2}(X)$. Since the maximal spectral type of $T$ is continuous and $P(z)$ is analytic we have

$$
\text { ker } P(T)=\{0\} \text {. }
$$

Thus $J f=0$ for each $f \in L_{0}^{2}(X)$ i.e., $J$ is trivial and the proof of the lemma is complete.

Corollary 2. The staircase maps with bounded cutting parameter are spectrally disjoint from any mixing maps.

Proof. By the definition of staircase maps it is easy to prove that the sequence $T^{h_{n}}$ converges weakly to $\sum_{j=0}^{r-1} \frac{1}{r-1} T^{j}$ where $T^{0}$ is the identity map.

One may apply Lemma 3 also to the "historical" Chacon map [6], given by

$$
p_{k}=2, k \in \mathbb{N}^{*} \text { and } a_{1}=1, a_{2}=0 .
$$

From the construction one may check that the sequence $\left(T^{h_{k}}\right)_{k \in \mathbb{N}^{*}}$ converges weakly to $\sum_{k=0}^{+\infty} \frac{1}{2^{k}} T^{k}$. Thus it is easy to get from Lemma 3 the following result. 
Corollary 3. The historical Chacon map is spectrally disjoint from any mixing map.

Remark 2. Combining the same methods with the continuous version of Beurling theorem we can extend Theorem 8 to flows. Now, applying the results of Frączek-Lemańczyk [12], [13], we can exhibit examples of maps for which the conditions of the theorem are satisfied.

\section{Mathew-NADKARNi TRANSFORMation}

In this section we show that $\alpha$-rigidity alone is not enough to ensure the spectrum is singular. Indeed, we will show that the Mathew-Nadkarni transformation is an example of $\frac{1}{2}$-rigid transformation whose spectrum has a Lebesgue component.

The Mathew-Nadkarni transformation is a $\mathbb{Z}_{2}$-extension of the odometer or von Neumann-Kakutani adding machine $\tau^{1}, \mathbb{Z}_{2} \stackrel{\text { def }}{=}\{0,1\}$ endowed with its Haar measure $h \stackrel{\text { def }}{=} \frac{1}{2} \delta_{0}+\frac{1}{2} \delta_{1}$. Explicitly, define $T_{\phi}:[0,1) \times \mathbb{Z}_{2} \mapsto[0,1) \times \mathbb{Z}_{2}$ by $T_{\phi}(x, g)=(T x, \phi(x)+g)$, where $T$ is the adding machine.

$T$ is defined by mapping the interval $\left[1-\frac{1}{2^{n}}, 1-\frac{1}{2^{n+1}}\right)$ linearly onto the interval $\left[\frac{1}{2^{n+1}}, \frac{1}{2^{n}}\right), n=0,1,2 \cdots$. Note that $T$ is a rank one transformation which is also easily described using cutting and stacking. The cocycle $\phi$ is defined (inductively over all "levels" of the tower associated to $T$ except the last one) to be 0 on the intervals $\left[1-\frac{1}{2^{n}}, 1-\frac{1}{2^{n}}+\frac{1}{2^{n+2}}\right)$, and 1 on the intervals $\left[1-\frac{1}{2^{n}}+\frac{1}{2^{n+2}}, 1-\frac{1}{2^{n+1}}\right)$, $n=0,1,2 \cdots$..

Theorem 10 (Mathew-Nadkarni). The Mathew-Nadkarni transformation has a spectrum consisting of a Lebesgue component with multiplicity 2, together with discrete component.

The proof can be found in [19] or [31]. We will proof the following theorem.

Theorem 11. The Mathew-Nadkarni transformation is $\frac{1}{2}$-rigid.

Proof. Recall that the operator $U_{T_{\phi}}: L^{2}\left([0,1) \times \mathbb{Z}_{2}, \mu \otimes h\right) \rightarrow L^{2}\left([0,1) \times \mathbb{Z}_{2}, \mu \otimes h\right)$ has a direct sum decomposition

$$
L^{2}\left([0,1) \times \mathbb{Z}_{2}, \mu \otimes h\right)=L_{0} \oplus L_{1},
$$

where $L_{0}=\left\{f \otimes 1: f \in L^{2}([0,1))\right\}$ and $L_{1}=\left\{f \otimes \chi: f \in L^{2}([0,1))\right.$ and $\chi(g)=$ $(-1)^{g}$, for $\left.g \in \mathbb{Z}_{2}\right\}$.

Let $A$ be a Borel set and $\varepsilon \in \mathbb{Z}_{2}$ then

$$
\mathbb{I}_{A \times\{\varepsilon\}}=f_{1}+\chi f_{2},
$$

where $f_{1}=\frac{1}{2} \mathbb{I}_{A}, f_{2}=\chi(\varepsilon) f_{1}$. Let $\sigma_{d}$ be the discrete part of the spectral measure $\sigma_{\mathbb{I}_{A \times\{\varepsilon\}}}$. Then that there exists a sequence of integers $\left(n_{k}\right)$ such that

$$
\lim _{k \rightarrow \infty} \hat{\sigma}_{A \times\{\varepsilon\}}\left(n_{k}\right)=\lim _{k \rightarrow \infty} \hat{\sigma}_{d}\left(n_{k}\right)=\left(f_{1}, f_{1}\right)=\frac{1}{2} m \otimes h(A \times\{\varepsilon\}) .
$$

\footnotetext{
${ }^{1}$ see [23], [15] or [20].
} 
Hence, by a standard argument, for any Borel set $B$ in the $\sigma$-algebra of $[0,1) \times \mathbb{Z}_{2}$ we have

$$
\liminf _{k \rightarrow \infty} \hat{\sigma}_{B}\left(n_{k}\right) \geq \frac{1}{2} m \otimes h(B)
$$

This completes the proof of the theorem.

Remark 3. The Mathew-Nadkarni transformation is not weakly mixing but using the Ageev's construction [2] one can produce an $\alpha$-rigid transformations with continuous spectrum and Lebesgue component of even multiplicity. Let us remark also that Mathew-Nadkarni's construction contains continuum pairwise non-isomorphic dynamical systems [31]. It follows that one can produce a continuum of $\alpha$-rigid transformations with 2-fold Lebesgue spectrum.

V. V. Ryzhikov told us that Ageev had obtained $\alpha$-rigid maps with Lebesgue spectrum using the examples of Parry-Helson. This result has never been published. Ryzhikov also communicated us that Ageev used the same assertion as it was pointed out to us also by M. Lemanczyk:

If $T$ is rigid and the cocyle $\phi: X \longrightarrow \mathbb{Z}_{2}$ gives Lebesgue spectrum for $T_{\phi}$ (such $\phi$ exists over each rigid maps by Helson-Parry [18]), then $T_{\phi}$ is $\frac{1}{2}$-rigid.

\section{Substitution EXAmples}

In this section we review some examples of $\alpha$-rigid transformations whose spectrum has Lebesgue component. In particular we will give an example with $0<$ $\alpha \ll \frac{1}{2}$ coming from substitution theory.

A vast literature is devoted to substitutions, whose Bible is [33]. They appear as symbolic systems defined on a finite alphabet $A=\{0,1, \cdots, k-1\}$; a substitution $\xi$ is a mapping from $A$ to the set $A^{*}$ of all finite words of A. It extends naturally into a morphism of $A^{*}$ by concatenation. We restrict ourselves to the case when $\xi(0)$ begins with 0 and the length of $\xi^{n}(0)$ tends to infinity with $n$. The infinite sequence $u$ beginning with $\xi^{n}(0)$ for all $n$ is then called a fixed point of $\xi$ and the symbolic system associated to $u$ is called the dynamical system associated to $\xi$.

When $\xi$ is primitive, (i.e., there exists $n$ such that $\alpha$ appears in $\xi^{n}(\beta)$ for all $\alpha, \beta \in$ $A$ ), the system is uniquely ergodic, and we can consider the measure-preserving system associated to $\xi$, to which we refer in short by "the substitution $\xi$ ". The composition matrix $M$ of $\xi$ is the matrix whose entries are $\ell_{i j}=O_{i}(\xi(j))$, where $i, j \in A$ and $O_{i}(\xi(j))$ is the number of $i^{\prime}$ s occurring in $\xi(j)$. If $\xi$ is primitive, it follows from the Perron-Frobenius theorem that $M$ admits a strictly positive simple eigenvalue $\theta$, such that $\theta>|\lambda|$, for any other eigenvalue $\lambda$ and there exists a strictly positive eigenvector corresponding to $\theta$. It is easy to see that (see [33]), for any $a \in A$, the sequence of $k$-dimensional vector

$$
\left(\frac{O_{0}\left(\xi^{n}(a)\right)}{\theta^{n}}, \cdots, \frac{O_{k}\left(\xi^{n}(a)\right)}{\theta^{n}}\right),
$$

converges to a strictly positive eigenvector $v(a)$ corresponding to $\theta$.

A classical result on primitive substitution is the Keane-Dekking theorem [8]. They proved that the dynamical system arising from a primitive substitution is not mixing. Their proof, with the above notations, contains the following. 
Theorem 12. The dynamical system arising from a primitive substitution $\xi$ is $r \rho$-rigid. Here $r$ is the maximum of the measure of the cylinders set $[a a], a \in A$, and $\rho$ is the $\ell_{1}$-norm of the strictly positive eigenvector $v\left(a_{r}\right)$ corresponding to the Perron-Frobenius eigenvalue $\theta$ of $\xi, a_{r}$ is a letter for which the measure of $\left[a_{r} a_{r}\right]$ is $r$.

Actually it is obvious to compute the constant $r$. In fact, let $\xi_{2}$ be the substitution defined on the alphabet $A_{2}=\{(a b), a, b \in A\}$ in the following way :

$$
\text { if } \xi(a b)=\xi(a) \xi(b)=y_{0} y_{1} y_{2} y_{3},
$$

then we set

$$
\xi_{2}(a b)=\left(y_{0} y_{1}\right)\left(y_{1} y_{2}\right) .
$$

Now it is easy to compute the normalized positive eigenvector corresponding to the dominant eigenvalue of the composition matrix $M_{2}$ of $\xi_{2}$.

One of the classical examples of substitutions is the Rudin-Shapiro substitution. This is defined on the alphabet $A=\{0,1,2,3\}$ in the following way

$$
\begin{array}{ll}
\xi(0)=02, & \xi(1)=32, \\
\xi(2)=01, & \xi(3)=31 .
\end{array}
$$

M. Queffélec in [32] shows that the continuous part of the Rudin-Shapiro dynamical system is Lebesgue with multiplicity 2 and it is easy to prove that the Rudin-Shapiro dynamical system is $\frac{1}{2}$-rigid. Another example of a substitution with Lebesgue spectrum is given by the following substitution $\xi$ on the alphabet $A=\{0,1,2\}$ :

$$
\begin{aligned}
& \xi(0)=001 \\
& \xi(1)=122 \\
& \xi(2)=210 .
\end{aligned}
$$

This substitution has Lebesgue spectrum with multiplicity 2 in the orthocomplement of eigenfunctions [33, p. 221]. We point out that one may use a standard computer program to compute approximatively the constant of $\alpha$-rigidity of $\xi$ which is approximatively equal to $0.3104979673 \times 10^{-7}$.

Acknowledgment 1. I would like to express my thanks to Late J. De Sam Lazaro 2 , J-P. Thouvenot and M. Disertori for their considerable help and encouragement. I'm greatly indebted to V. V. Ryzhikov for the proof of Baxter theorem and for pointing out to me Lemma 3. Finally I wish to express my heartfelt gratitude to F. Parreau for his collaboration in proving Theorem 10 and to M. Lemańczyk for stimulating conversations on the subject and the many suggestions for applications of our main result.

\footnotetext{
${ }^{2}$ This paper was prepared under the supervision of professor José De Sam Lazaro two years before his death.
} 


\section{REFERENCES}

[1] E. H. el Abdalaoui, A. Nogueira, T. de la Rue, Weak mixing of maps with bounded cutting parameter. New York J. Math., 11, (2005), 81-87 (electronic).

[2] O. N. Ageev, Dynamical systems with an even-multiplicity Lebesgue component in the spectrum, Math. USSR. Sbornik., Vol 64 (2), (1989), 305-316.

[3] P. Arnoux, D. Ornstein, B. Weiss, Cutting and stacking, interval exchanges and geometric models, Isr. J. Math., Vol 50 (1-2), (1985), 160-168.

[4] A. Beurling, Quasianalyticity and General Distributions, Stanford Univ. Lect. Notes, no. 30, 1961.

[5] J. R. Baxter, On the Class of Ergodic Transformations, PhD Thesis, University of Toronto, 1969.

[6] R. V. Chacon, Weakly mixing transformations which are not strongly mixing, Proc. Amer. Math. Soc., 22 (1969), 559-562.

[7] R. V. Chacon, Transformations having continuous spectrum, J. Math. Mech., 16 (1966), 399-415.

[8] F. M. Dekking and M. Keane, Mixing properties of substitutions, Zeit. Wahr., 42 (1978), 23-33.

[9] A. del Junco and M. Lemańczyk, Generic spectral properties of measure-preserving maps and applications, Proc. Am. Math. Soc., 115 (1992), 725-736.

[10] A. del Junco and D. Rudolph, On ergodic actions whose self-joinings are graphs, Ergodic. Th and Dynam. Sys., 7, (1987), 531-557.

[11] A. del Junco, A. Rahe, L. Swanson, Chacon's automorphism has minimal self-joinings, J. Anal. Math.,37 (1980), 276-284.

[12] K. Fraçzek, M. Lemańczyk, On disjointness properties of some smooth flows, Fund. Math., 185 (2005), no. 2, 117-142.

[13] K. Fraçzek, M. Lemańczyk, A class of special flows over irrational rotations which is disjoint from mixing flows, Ergodic Theory Dynam. Systems, 24 (2004), no. 4, 10831095.

[14] N. Friedman , Partial mixing, partial rigidity, and factors, Contemp. Math.,94, Collection: Measure and measurable dynamics (Rochester, NY, 1987), 141-145.

[15] N. A. Friedman, Introduction to Ergodic Theory, Van Nostrand Reinhold, New York, 1970.

[16] N. A. Friedman, Replication and stacking in ergodic theory, Amer. Math. Monthly, 99 (1992), 31-34

[17] M. Guenais, Singularité des produits de Anzai associs aux fonctions caractristiques d'un intervalle. Bull. Soc. Math. France, 127 (1999), no. 1, 71-93.

[18] H. Helson and W. Parry, Cocycles and spectra, Arkiv Math., 16 (1978), 195-206.

[19] J. Mathew and M. G. Nadkarni, A measure-preserving transformation whose spectrum has a Lebesgue component of multiplicity two, Bull. Lond. Math. Soc., 16 (1984), 402-406.

[20] M. G. Nadkarni, Spectral Theory of Dynamical Systems , Birkhäuser Advanced Texts: Basel Textbooks, Birkhäuser Verlag, Basel, 1998.

[21] V. I. Oseledets, On the spectrum of ergodic automorphisms, Doklady Akad. Nauk SSSR, 168, 5 (1966),1009-1011 (in Russian), translated in Soviet Math. Doklady, 7 (1966), 776-779.

[22] W. Parry, Topics in Ergodic Theory , Cambridge University Press, 1981.

[23] K. Petersen, Ergodic Theory, Cambridge University Press, 1983.

[24] A. A. Prikhod'ko and V. V. Ryzhikov, Disjointness of the convolutions for Chacon's automorphism, Dedicated to the memory of Anzelm Iwanik. Colloq. Math., 84/85 (2000), part 1, 67-74.

[25] T. Kamae, Spectral properties of automata generating sequences, Unpublished.

[26] A. B. Katok, Constructions in Ergodic Theory, preprint.

[27] A. B. Katok, Interval exchange transformations and some special flows are not mixing, Isr. J. Math., Vol 35, 4, (1980), 301-310.

[28] A. B. Katok, A. M. Stepin, Approximation of ergodic dynamic systems by periodic transformations, Dokl. Akad. Nauk SSSR, 171 (1966), 1268-1271; Soviet Math. Dokl., 7 (1966), 1638-1641. 
[29] J. L. King and J-P. Thouvenot, A canonical structure theorem for joining-rank maps, J. Analyse Math., 51 (1988), 182-227.

[30] I. Klemes \& K. Reinhold, Rank one transformations with singular spectre type, Isr. J. Math.,vol 98, (1997), 1-14.

[31] M. Lemańczyk, Toeplitz $\mathbb{Z}_{2}$-extensions, Ann. Inst. Henri Poincaré, vol 24, $\mathrm{n}^{\circ} 1$, (1988), 1-43.

[32] M. Queffélec, Une nouvelle propriété des suites de Rudin-Shapiro $t$ Ann. Fourier, Grenoble, 37, 2 (1987), 115-138.

[33] M. Queffélec, Substitution Dynamical Systems-Spectral Analysis, In A. Dold and B. Eckmann, editors, Lecture notes in Mathematics, vol 1294, Spring-Verlag, 1987.

[34] W. Rudin, Fourier Analysis on Groups, Interscience Tracts in Math. 12. J. Wiley ans sons, 1962.

[35] E. A., Jr. Robinson, Ergodic measure preserving transformations with arbitrary finite spectral multiplicities, Invent. Math., 72 (1983), no. 2, 299-314.

[36] V. A. Rokhlin, Selected topics in the metric theory of dynamical systems, Uspekhi Mat. Nauk." New series", 4 (1949), 57-128 (Russian); Amer. Math. Soc. Transl., 2, 40 (1966), 171-240, 1979, 97-122.

[37] D. Rudolph, An Example of a measure-preserving map with minimal self-joining and applications, J. analyse Math., 35, 1979, 97-122.

[38] V. V. Ryzhikov, Joinings, Intertwining operators, factors and mixing properties of dynamical systems, Russian. Acad. Sci. Izv. Math., Vol 26, (1994), 91-114.

[39] V. V. Ryzhikov, Around simple dynamical systems. induced joinings and multiple mixing J. Dynam. Control Systems, 3 (1997), no. 1, 111-127.

[40] V. V. Ryzhikov, Stochastic intertwinings and multiple mixing of dynamical systems, J. Dynam. Control Systems, 2 (1996), no. 1, 1-19.

[41] V. V. Ryzhikov, Prive communication.

[42] A. M. Stepin, Spectral properties of generic dynamical system, Math. U.S.S.R. Izv., 50 (1986), 159-192.

[43] W. A. Veech, The metric theory of interval exchange transformations. I Generic spectral properties, Amer. J. Math., 106 (1984), 1331-1359.

[44] W. A. Veech, A criterion for a process to be prime, Monatsh. Math., 94 (1982), $335-341$.

(received version 10.10.2007)

(revised version 04.01.2009)

Author's address:

E. H. El Abdalaoui

Department of Matehmatics, LMRS UMR 6085 CNRS,

University of Rouen,

Avenue de l'Université, BP.12, 76801 Saint Etienne du Rouvray, France

E-mail:elhoucein.elabdalaoui@univ-rouen.fr 